\title{
Clinical Features and Management of a Median Cleft Lip
}

\author{
Kyung S. Koh, Do Yeon Kim, Tae Suk Oh \\ Department of Plastic Surgery, Asan Medical Center, University of Ulsan College of Medicine, Seoul, Korea
}

Background Median cleft lip is a rare anomaly consisting of a midline vertical cleft through the upper lip. It can also involve the premaxillary bone, the nasal septum, and the central nervous system. In our current report, we present the clinical features of 6 patients with a median cleft lip and their surgical management according to the accompanying anomalies. Methods From December 2010 to January 2014, 6 patients with a median cleft lip were reviewed. Five of these cases underwent surgical correction; alveolar bone grafting was performed in a patient with a median alveolar cleft. The surgical technique included inverted-U excision of the upper lip and repair of the orbicularis oris muscle. The mean follow-up period was 20.4 months (range, 7.4-44.0 months).

Results The study patients presented various anomalous features. Five patients received surgical correction, 4 with repair of the median cleft lip, and one with iliac bone grafting for median alveolar cleft. A patient with basal sphenoethmoidal meningocele was managed with transoral endoscopic surgery for repair of the meningocele. Successful surgical repair was achieved in all cases with no postoperative complications.

Conclusions Relatively mild forms of median cleft lip can be corrected with inverted-U excision with good aesthetic outcomes. In addition, there is a broad spectrum of clinical features and various anomalies, such as nasal deformity, alveolar cleft, and short upper frenulum, which require close evaluation. The timing of the operation should be decided considering the presence of other anomalies that can threaten patient survival.

\section{Keywords Frontonasal dysplasia / Median facial cleft syndrome / Median cleft lip}

Received: 12 Jan $2016 \bullet$ Revised: 13 Apr $2016 \bullet$ Accepted: 14 Apr 2016

pISSN: 2234-6163 • elSSN: 2234-6171 • http://dx.doi.org/10.5999/aps.2016.43.3.242 • Arch Plast Surg 2016;43:242-247

\author{
Correspondence: Kyung S. Koh \\ Department of Plastic Surgery, \\ Asan Medical Center, University of \\ Ulsan College of Medicine, \\ 88 Olympic-ro 43-gil, Songpa-gu, \\ Seoul 05505, Korea \\ Tel: +82-2-3010-3600 \\ Fax: +82-2-476-7471 \\ E-mail: kskoh@amc.seoul.kr
}

No potential conflict of interest relevant to this article was reported.

\section{INTRODUCTION}

A median cleft lip is an anomaly that presents with a congenital vertical cleft through the center of the upper lip. The incidence has been reported to be $0.43 \%-0.73 \%$ in the cleft lip population and 1:1,000,000 in the general population $[1,2]$. The developmental origin of a median cleft of the upper lip is unclear. However, it is caused by malfusion of the medial nasal prominences. It can also involve the premaxillary bone, the nasal septum, and the central nervous system, causing flat nose, median alveolar cleft, hypotelorism or hypertelorism, monophthalmia, and proboscis. The spectrum of median cleft lip varies from a simple central vermillion notch to a wide complete cleft.

As described by DeMyer, there are two separate groups of facial anomaly, associated with either orbital hypotelorism or hypertelorism [3]. Reports have been made in the literature, however, about rare cases of incomplete median cleft lip with normotelorism. These cases have relatively mild cleft lip associated 
with other anomalies that can be corrected surgically. There have been few reports on median cleft lip and its surgical management. Thorough evaluation of the various forms of accompanying anomalies is mandatory in median cleft lip patients. If left untreated, some accompanying anomalies can lead to severe developmental impairment. Thus, management of such anomalies should be taken into consideration before or after the surgical correction of the median cleft lip. In our current study, we observed the clinical features of median cleft lip cases and their surgical management, including the accompanying anomalies.

\section{METHODS}

Six patients who received treatment for median cleft lip from December 2010 to January 2014 at our hospital were included. All of these cases, except for one patient with basal sphenoethmoidal meningocele, underwent surgical correction. The age of the patients on the date of surgical management ranged from 82 days to 9 years. The mean follow up period was 20.4 months (ranging from 7.4 months to 44.0 months).

The surgical technique consisted of inverted- $U$ excision of the upper lip. General anesthesia with oral intubation was performed, followed by an inverted- $U$ design right above the mucocutaneous white roll (Fig. 1A). The correction of the upper lip frenulum was planned (Fig. 1B). An incision was performed, followed by inverted-U excision of the skin. The orbicularis oris muscle was identified and repaired with Vicryl suturing (Fig. 2A), followed by dermal and skin closure (Fig. 2B). In the single case in our series with median alveolar cleft, cancellous bone harvested from the iliac bone was grafted.

\section{RESULTS}

From December 2010 to January 2014, there were 6 median

\section{Fig. 1. Design for the cleft lip repair}

(A) Design for inverted-U incision. The curvature of the incision line allows the skin lengthened after closure to yield a longer philtral column. (B) Design extended to the mucosal side and upper lip frenulum.
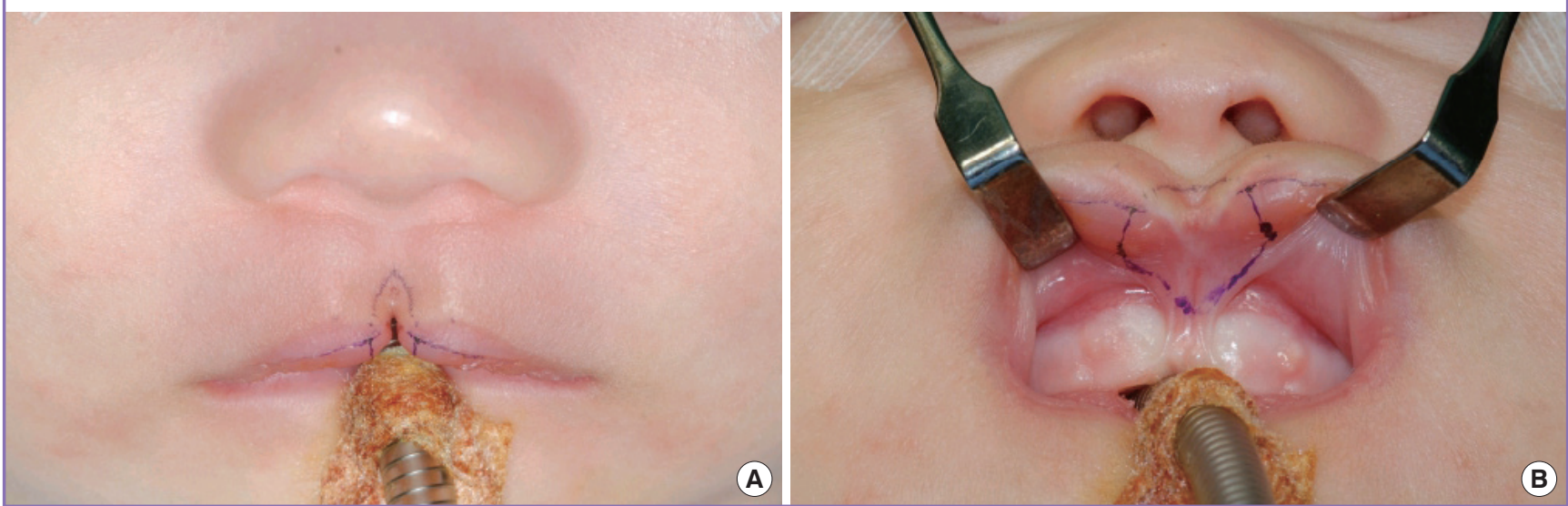

\section{Fig. 2. Surgical method of the cleft lip repair}

(A) After dissection of the orbicularis oris muscle, reorientation and repair with 4-0 Vicryl sutures at 3 points were done and the pars marginalis was sutured with 6-0 polydioxanone (PDS) suture at 1 point. (B) Closure of the skin and mucosa with a natural Cupid's bow was achieved.
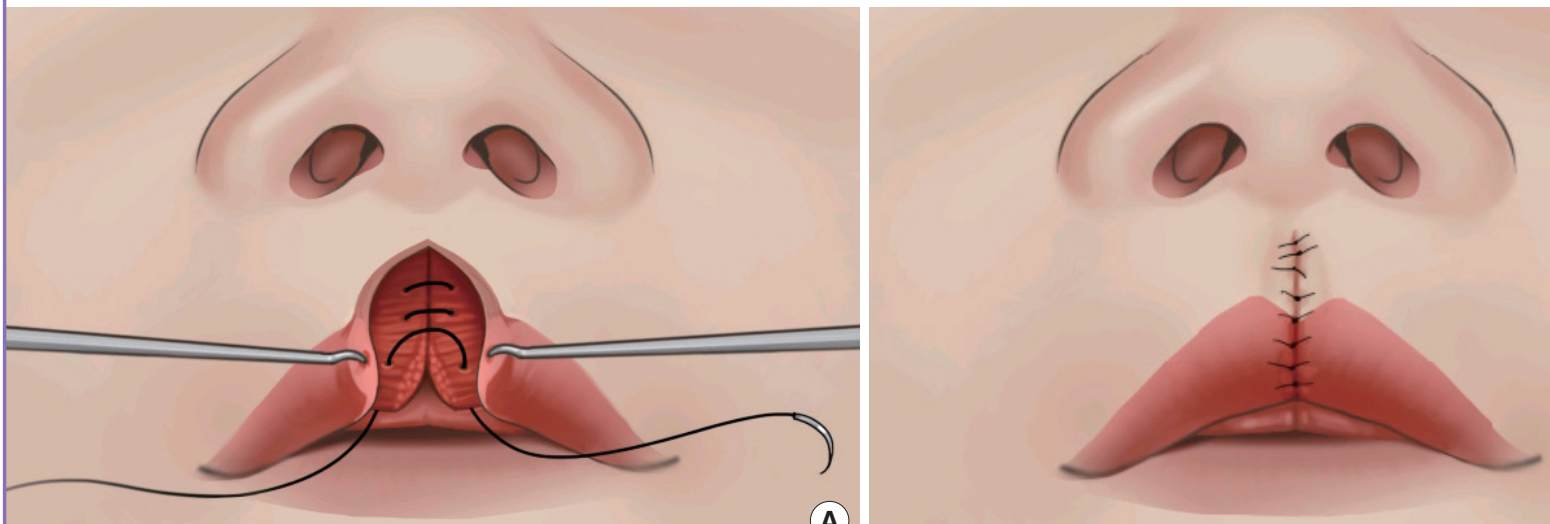
Table 1. Accompanying anomalies in each of the cases

\begin{tabular}{|c|c|c|c|c|c|c|c|}
\hline Age & Sex & Alveolar cleft & Wide columella & $\begin{array}{l}\text { Short upper lip } \\
\text { frenulum }\end{array}$ & $\begin{array}{l}\text { Upper } \\
\text { lip pit }\end{array}$ & $\begin{array}{c}\text { Basal } \\
\text { sphenoethmoidal } \\
\text { meningocele }\end{array}$ & $\begin{array}{l}\text { Colobomatous } \\
\text { microphthalmia }\end{array}$ \\
\hline 86 day & Female & - & 0 & 0 & - & - & - \\
\hline 91 day & Female & 0 & - & - & - & - & - \\
\hline 83 day & Female & 0 & - & $\bigcirc$ & - & - & - \\
\hline 82 day & Male & 0 & - & 0 & 0 & - & - \\
\hline $9 \mathrm{yr}$ & Female & 0 & - & - & - & - & - \\
\hline 85 day & Female & - & 0 & - & - & 0 & 0 \\
\hline
\end{tabular}

\section{Fig. 3. Median cleft lip before and after surgery}

(A) Preoperative feature of median cleft lip. (B) Outcome of surgery at 4 months postoperatively. A natural and symmetric Cupid's bow was achieved with inverted-U excision followed by three-layered closure with muscle repair.

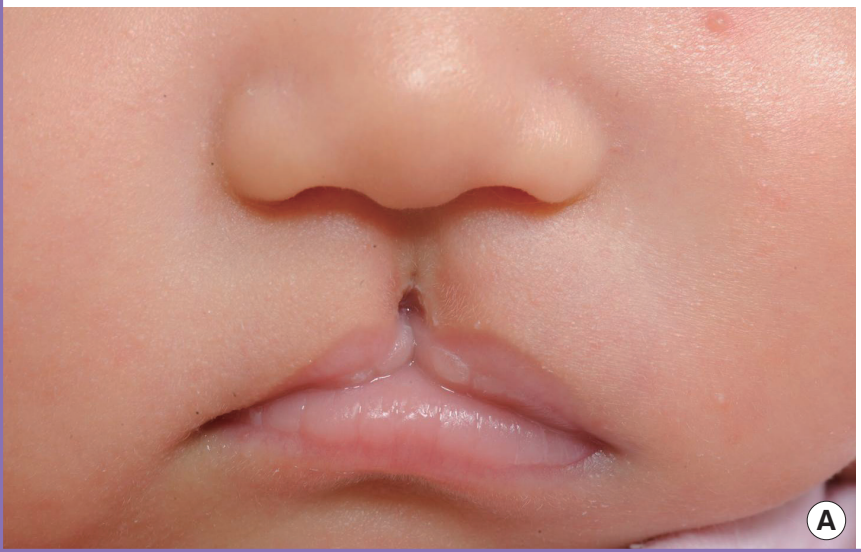

cleft lip patients treated at our institution with various spectra of anomalous features (Table 1), 5 of whom received surgical correction. Five of these patients were female. One of these cases was a 9-year-old female patient with an alveolar cleft on the midline of the maxillary wall who had previously undergone surgical repair for median cleft lip and palatoplasty for a cleft palate. In this patient, iliac bone grafting was performed to correct the anterior maxilloalveolar bone defect. This patient also presented secondary notching of the upper lip vermilion on the midline, which was managed with free border correction. Another patient with basal sphenoethmoidal meningocele was managed with transoral endoscopic surgery for repair of the meningocele, and the repair of the median cleft lip was delayed. One patient with accompanying upper lip pit on the midline underwent pit removal together with cleft repair. Surgical repair was performed successfully in all cases and there were no specific complications such as infection, dehiscence, or hypertrophic scar.

\section{Case 1}

Case 1 was a patient with a median cleft lip who received surgical correction 86 days after birth. A wide columella and a visible stria on the bulbous nasal tip were observed. Inverted-U incision

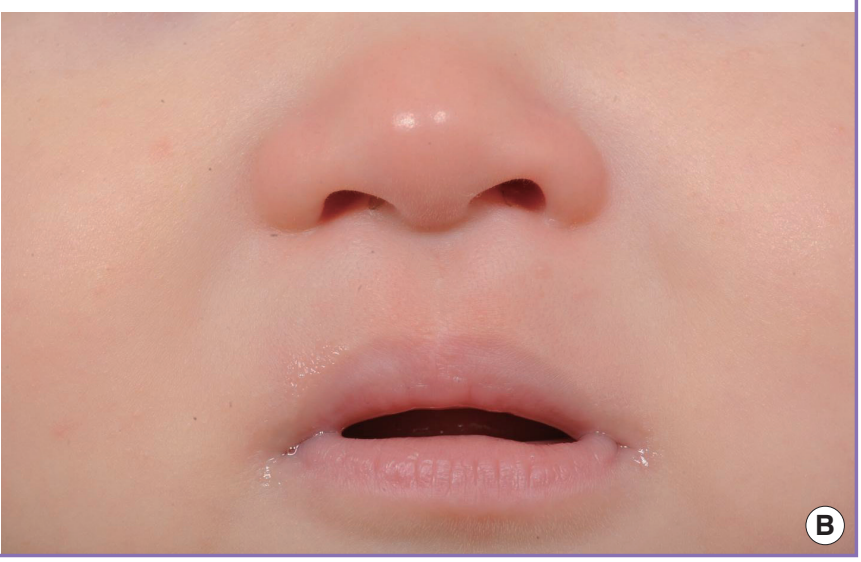

was performed. Dissection and excision were followed by orbicularis muscle reorientation and repair. Upper lip frenulotomy was also performed.

\section{Case 2}

This patient received surgical correction for a median cleft lip at 91 days after birth (Fig. 3). A wide columella and deficient alveolar ridge were observed. The correction was performed on the upper lip, including the orbicularis oris muscle.

\section{Case 3}

This patient received surgical correction 83 days after birth (Fig. 4). A wide columella and median alveolar cleft were observed. Correction of the median cleft and muscle repair were performed. The upper lip frenulum was excised.

\section{Case 4}

This patient received surgical correction 82 days after birth. A median alveolar cleft and sinus extending to the premaxilla were observed on the upper lip (Fig. 5A). The median cleft was corrected (Fig. 5B) and the sinus was excised with a probe to the pit. The short upper lip frenulum was excised. 


\section{Fig. 4. Another case of median cleft lip}

(A) Preoperative feature of median cleft lip. (B) Outcome of surgery at 9 months postoperatively. A natural Cupid's bow, philtrum and tubercle was achieved.

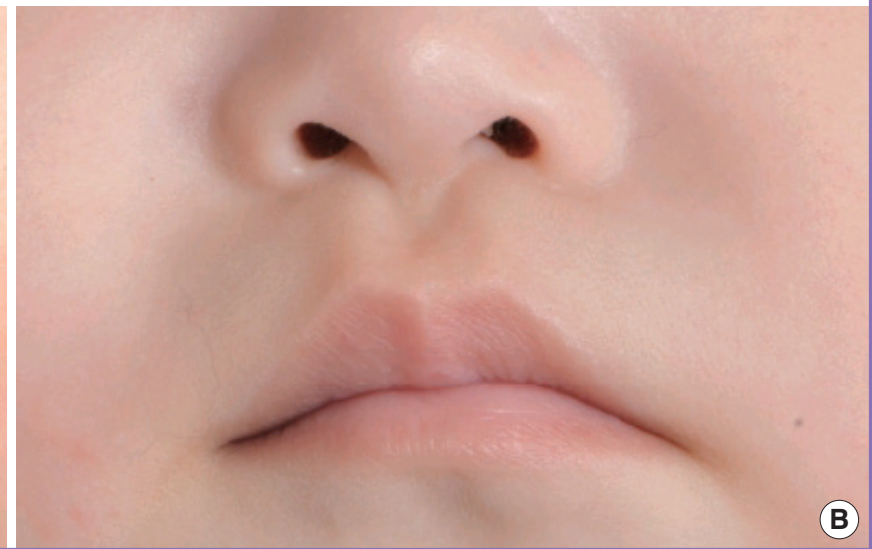

\section{Fig. 5. Median cleft lip with upper lip pit}

(A) Preoperative feature of median cleft lip and upper lip pit. (B) Repaired median cleft lip with pit removal. Natural, symmetrical Cupid's bow was achieved postoperatively and successful excision of the accompanying upper lip pit was done.

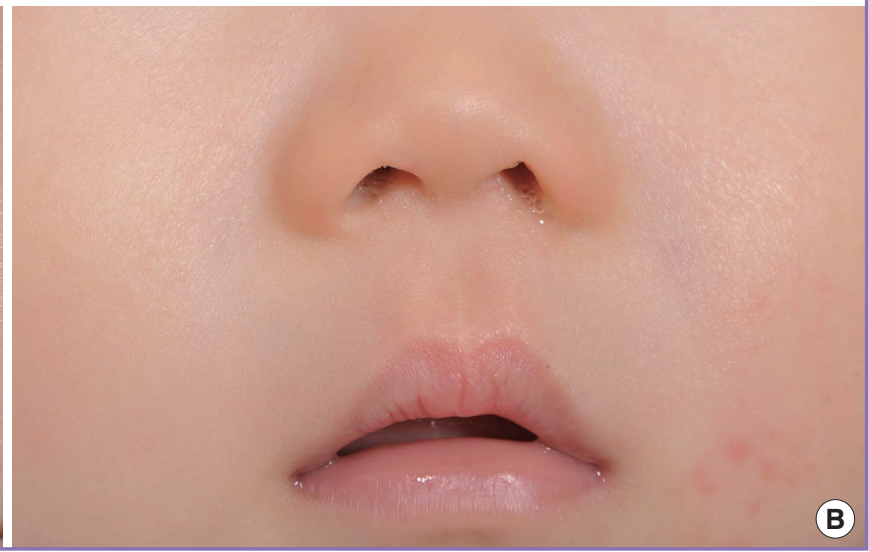

\section{Case 5}

Case 5 was a 9-year-old patient with a median cleft lip who received surgical correction of the anterior maxilloalveolar bone deformity (Fig. 6A, B). Presurgical orthodontic treatment was administered to expand the palatal arch, and several months of additional orthodontic treatment enabled incisor approximation. A midline defect of the maxillary wall and alveolus was observed on preoperative computed tomography (CT) with three dimensional (3D)-reconstruction (Fig. 6C). Cancellous bone was harvested from the iliac bone. Bone grafting and free border correction of the secondary vermilion notching was performed. Postoperative CT with 3D reconstruction confirmed successful bone grafting on the midline (Fig. 6D).

\section{Case 6}

Case 6 was born with a median cleft lip and palate. A midline cystic mass was observed through the cleft palate. Basal spheno- ethmoidal meningocele was observed. This patient received transoral endoscopic surgery for repair of the encephalocele 85 days after birth. After this surgery, repair of the median cleft lip and palate was considered. However, the patient was lost to follow-up.

\section{DISCUSSION}

A median cleft lip is defined as a vertical cleft through the center of the upper lip. Its incidence is reported to be about $0.43 \%-0.73 \%$ of the craniofacial cleft population [2]. There have been some reports in the literature describing different variations of the median cleft lip. Midline facial defects can be traced to a period corresponding to the third week of gestation. During this time, the primitive forebrain shows bilateral thickenings of the frontonasal process. These thickenings of the ectoderm emerge as the lateral and medial nasal prominences. Fusion of the inferior me- 


\section{Fig. 6. Bone grafting to a median alveolar cleft}

(A) Dental view of preoperative median alveolar cleft after presurgical orthodontic treatment. (B) Outcome at 13 months after bone grafting. Further orthodontic treatment for incisor approximation was done for several months. (C) Three dimensional (3D)-computed tomography (CT) image of preoperative median alveolar cleft. (D) Postoperative image to identify successful bone grafting. 3D-reconstructed CT image confirmed successful iliac bone grafting compared to the preoperative image shown in (C).
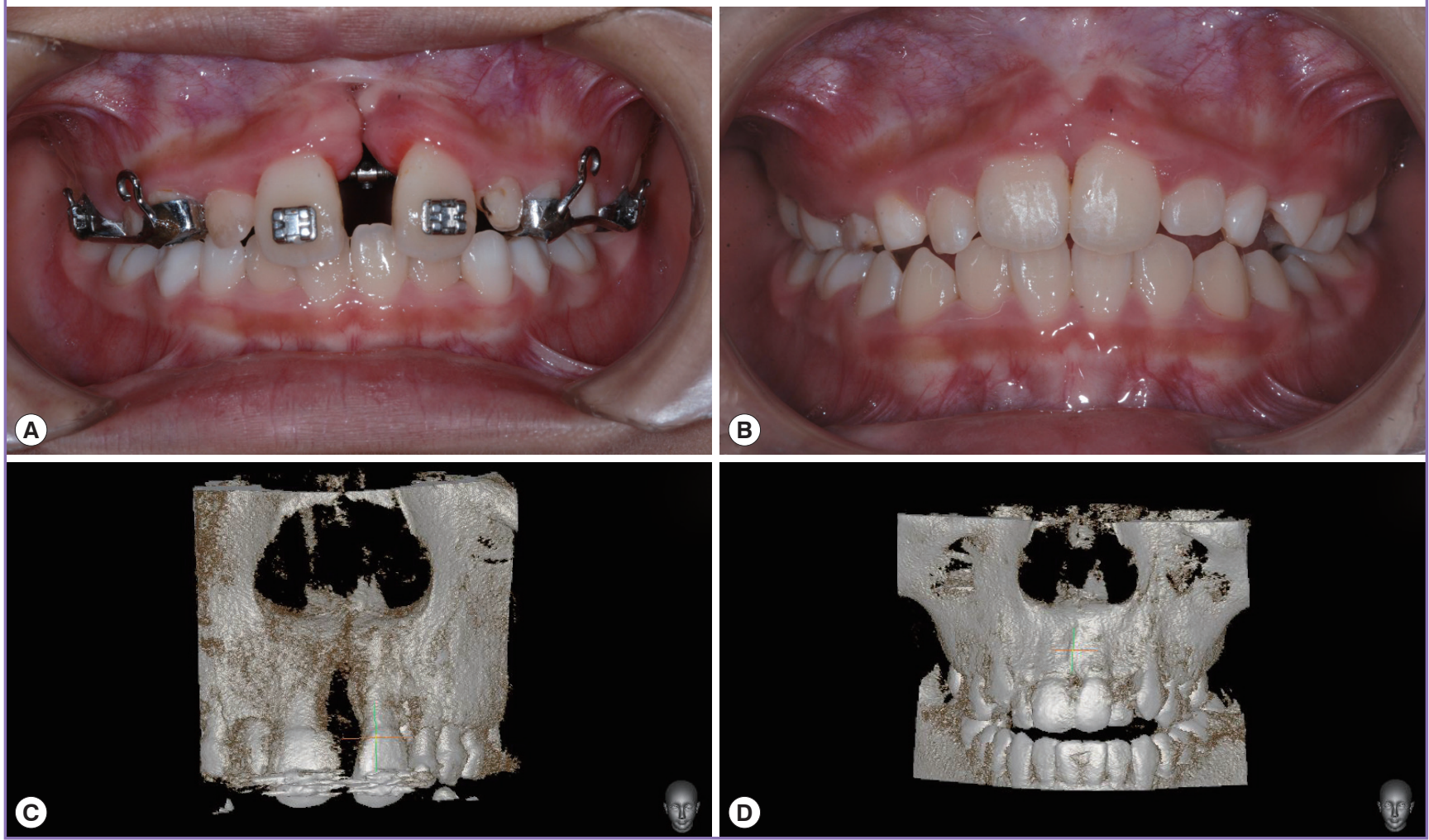

dial nasal prominence, the lateral prominence, and the medial aspect of the maxillary process of the first visceral arch results in normal development of the upper lip [4,5]. Failure of this developmental stage can lead to a duplication or widening of the midline structures, or hypoplasia/agenesis of the structures with or without associated anomalies such as bifurcation of the nose, hypertelorism, and cranial malformations. In our current report, we included patients with a median cleft of the upper lip, regardless of the presence of various forms of deformity of the nose, philtrum, or alveolus.

There has been one previous report on a surgical method to lengthen the skin in the center of the Cupid's bow using inverted-V excision [6]. In our current study cases, the median cleft lip could be surgically corrected using inverted-U excision to achieve natural alignment of the Cupid's bow. All of our study patients had an incomplete cleft lip. Intraoperatively, the orbicularis oris muscle fibers were interrupted. It has been shown previously that three-layered closure of the cleft lip after inverted-U excision requires plication or repair of the muscle $[7,8]$. As recommended by Millard, a combination of an inverted-V excision and a 90-degree angle in the excision $2 \mathrm{~mm}$ above the mucocu-

taneous white roll on each side of the cleft results in lengthening of the skin on the midline of the Cupid's bow. This technique also reduces the philtrum transversely; therefore, a natural appearance of the Cupid's bow can be achieved. Accurate design of the skin excision enables a natural postoperative contour of the Cupid's bow with sufficient tissue on both halves of the cleft lip. In our current cases, lengthening of the midline skin was achieved with inverted-U incision right above the mucocutaneous white roll. Excessive excision of the skin can lead to hypertrophic scarring, whereas insufficient excision can lead to an unnatural depression on the midline of the philtrum. With an approach that accurately determines the amount of skin to be removed, a natural alignment of the philtrum and Cupid's bow can be achieved.

In our current study cases, 4 patients had accompanying alveolar cleft. Radiographic imaging for the diagnosis of the alveolar defect and accompanying bone defects should be performed in such cases, and long-term follow-up of the bone defect for treatments including alveolar bone grafting is required. When indicated, iliac bone grafting with a conventional method can manage the bone defect [9] and preoperative/postoperative evaluation can be supplemented using 3-dimensionally reconstructed 
CT imaging, as done in our study patients. Preoperative and postoperative orthodontic treatment can be done for incisor approximation and alignment. There have been debates on the optimal timing of the bone grafting. This issue thus remains open for further investigation, despite the difficulty in doing so due to the rare incidence of a median cleft lip.

More severe forms of median cleft lip seem to accompany other anomalies and threaten the development and survival of the affected patients. When indicated, surgical treatment is mandatory for such anomalies prior to the repair of the cleft lip. Close follow-up and evaluation on the developmental status in addition to the structural anomalies of the patient should therefore be done. Our current study findings indicate that there are surgically correctable features and anomalies in median cleft lip patients. The timing of the operation should be decided according to the priority of correction, taking into consideration the various anomalies associated with the survival of these patients.

\section{REFERENCES}

1. Urata MM, Kawamoto Jr HK. Median clefts of the upper lip: a review and surgical management of a minor manifestation. J Craniofac Surg 2003;14:749-55.

2. DeMyer W. The median cleft face syndrome: differential diagnosis of cranium bifidum occultum, hypertelorism, and median cleft nose, lip, and palate. Neurology 1967;17:96171.

3. Apesos J, Anigian GM. Median cleft of the lip: its significance and surgical repair. Cleft Palate Craniofac J 1993;30:94-6.

4. Ichida M, Komuro Y, Yanai A. Consideration of median cleft lip with frenulum labii superior. J Craniofac Surg 2009;20: 1370-4.

5. Mazzola RF. Congenital malformations in the frontonasal area: their pathogenesis and classification. Clin Plast Surg 1976;3:573-609.

6. Millard DR. Median cleft lip with hypertelorism. In: Millard DR, editor. Cleft craft. the evolution of its surgery: bilateral and rare deformities 2 2. Boston: Little, Brown; 1977. p.72768.

7. Patel NP, Tantri MD. Median cleft of the upper lip: a rare case. Cleft Palate Craniofac J 2010;47:642-4.

8. Jian XC, Zheng L, Xu P, et al. Median cleft of the upper lip associated with a mass: a rare case. J Craniomaxillofac Surg 2014;42:1557-61.

9. Liao HT, Chen $\mathrm{CH}$, Bergeron L, et al. Alveolar bone grafting in the treatment of midline alveolar cleft and diastema in incomplete median cleft lip. Int J Oral Maxillofac Surg 2008; 37:886-91. 